\title{
Creating a Digital Health Smartphone App and Digital Phenotyping Platform for Mental Health and Diverse Healthcare Needs: an Interdisciplinary and Collaborative Approach
}

\author{
John Torous ${ }^{1} \cdot$ Hannah Wisniewski ${ }^{1} \cdot$ Bruce Bird $^{2} \cdot$ Elizabeth Carpenter ${ }^{3} \cdot$ Gary David $^{4} \cdot$ Eduardo Elejalde $^{5}$. \\ Dan Fulford ${ }^{6}$. Synthia Guimond ${ }^{7}$. Ryan Hays ${ }^{1}$. Philip Henson ${ }^{1}$ - Liza Hoffman ${ }^{1}$. Chun Lim ${ }^{8}$. Michael Menon ${ }^{9}$. \\ Valerie Noel ${ }^{10}$. John Pearson ${ }^{11}$ - Randy Peterson ${ }^{9}$ - Ammu Susheela ${ }^{11}$ - Haley Troy ${ }^{8}$ - Aditya Vaidyam ${ }^{1}$. \\ Emma Weizenbaum ${ }^{6}$. John A. Naslund ${ }^{12}$. Matcheri Keshavan ${ }^{1}$
}

Published online: 27 April 2019

(C) The Author(s) 2019

\begin{abstract}
As the potential of smartphone apps and sensors for healthcare and clinical research continues to expand, there is a concomitant need for open, accessible, and scalable digital tools. While many current app platforms offer useful solutions for either clinicians or patients, fewer seek to serve both and support the therapeutic relationship between them. Thus, we aimed to create a novel smartphone platform at the intersection of patient demands for trust, control, and community and clinician demands for transparent, data driven, and translational tools. The resulting LAMP platform has evolved through numerous iterations and with much feedback from patients, designers, sociologists, advocates, clinicians, researchers, app developers, and philanthropists. As an open and free tool, the LAMP platform continues to evolve as reflected in its current diverse use cases across research and clinical care in psychiatry, neurology, anesthesia, and psychology. In this paper, we explore the motivation, features, current progress, and next steps to pair the platform for use in a new digital psychiatry clinic, to advance digital interventions for youth mental health, and to bridge gaps in available mental health care for underserved patient groups. The code for the LAMP platform is freely shared with this paper to encourage others to adapt and improve on our team's efforts.
\end{abstract}

Keywords Mental health $\cdot$ Psychiatry $\cdot$ Digital health $\cdot$ Apps $\cdot$ Mobile health $\cdot$ mHealth

\section{Introduction}

The potential of digital mental health to increase access to high-quality and evidence-based mental healthcare continues to inspire investment, innovation, and progress. Whether measured by more than 10,000 mental health-related apps directly

John Torous

jtorous@bidmc.harvard.edu

1 Department of Psychiatry, Beth Israel Deaconess Medical Center, Harvard Medical School, Boston, MA, USA

2 Vinfen Community Mental Health Centers, Cambridge, MA, USA

3 Geisel School of Medicine, Dartmouth College, Hanover, NH, USA

4 Bentley University, Waltham, MA, USA

5 Natalia Mental Health Foundation, Coconut Grove, Miami, FL, USA

6 College of Health and Rehabilitation Sciences Sargent College, Boston University, Boston, MA, USA available for download today (Torous and Roberts 2017), exponential rise in academically published papers (Firth et al. 2016), or millions of dollars invested (Safavi et al. 2019), the field is clearly rapidly advancing (Hilty et al. 2017). Yet, actual uptake of mental health apps by patients (Fleming et al. 2018; Noel et al. 2018) and clinicians (Schueller et al. 2016)

7 The Royal's Institute of Mental Health Research, University of Ottawa, Ottawa, CA, USA

8 Department of Cognitive Neurology, Beth Israel Deaconess Medical Center, Harvard Medical School, Boston, MA, USA

9 Zco Corporation, Nashua, NH, USA

10 Westat, Inc., Lebanon, NH, USA

11 Department of Anesthesia, Beth Israel Deaconess Medical Center, Harvard Medical School, Boston, MA, USA

12 Department of Global Health and Social Medicine, Harvard Medical School, Boston, MA, USA 
remains low. This may be because the pace of innovation has pushed the digital mental health field ahead of national regulations for medical devices (McNiel and Binder 2018), medical-legal standards (Hilty et al. 2018a, b; Hsin and Torous 2018), privacy laws (Glenn and Monteith 2014), and even clinical evidence (Bhugra et al. 2017). A fitting and often referenced metaphor for this space is the 'wild west' (Anthes 2016), which captures the tension between expansion and innovation versus standards and reproducible evidence. While bringing order, trust, and clinical evidence into this space will happen, no single solution or correct approach exists (Torous and Haim 2018). One such approach is to focus on apps not as specific tools but rather customizable platforms to support both patient and clinician communities.

In this paper, we explore development of the LAMP (Learn Assess Manage and Prevent) platform as an example of codesigning an app and its supporting software ecosystem (platform) to meet the intersecting needs of clinical/research and patient/consumer communities. While the app may be the most visible part of any software platform, ensuring that there is a platform to support that app with storing, accessing, sharing, and analyzing the data is often overlooked when designing, supporting, and maintaining healthcare apps. And while many apps are built for a single purpose or to enable a single study, here we focus on creating tools to support diverse studies research as well as clinical care use cases. In order to create a platform designed for flexibility instead of an app created for a single purpose, we assembled a diverse team reflecting the input and experience of patients, clinicians, family members, researchers, app developers, designers, and donors. This paper explores the motivation, development, current progress, and next steps for the LAMP project. Its purpose is not to claim a solution but rather share our team's experiences and efforts to spark conversation about new approaches, encourage others to build off and improve on our work, and engage new stakeholders to join in the evolving digital health community.

\section{Unmet Needs for the Digital Mental Health Field}

Our project aimed to bridge three unmet needs around digital health platforms: (1) the need for translational apps with clinical relevance, (2) the need for open and reusable app platforms, and (3) the need to capture new types of data about interactions with the device, such as speed of answering questions, called metadata.

A first challenge is the need for health apps to offer proven clinical benefit to patients. Today numerous mental health apps exist both in the consumer and research space. The sheer volume and ease of availability of the over 10,000 patient facing apps does not equate to quality (Torous and Roberts 2017). Numerous review papers continue to raise caution about consumer apps offering interventions for depression (Firth et al. 2017a), bipolar disorder (Nicholas et al. 2015), anxiety disorders (Firth et al. 2017b), and more. As reflected in a constantly expanding Wikipedia page ("Mobile phone based sensing software" 2019), apps that do not offer interventions but offer real-time capture of environmental context with smartphone sensors (e.g., GPS, call/text logs), sometimes collectively referred to as digital phenotyping (Torous et al. 2016), can help track patient progress over time, identify triggers for relapse, and help quantify functional outcomes like sleep, physical activity, and social engagement (Mohr et al. 2017). Yet, few apps seek to bridge sensors/digital phenotyping and interventions, delivering both in a single platform able to detect risk and respond to it in real time. Thus, we sought to build an app platform, LAMP, that would prove translational between basic clinical studies, experimental therapeutics, and measurement-based care by offering resources for patients, researchers, and clinicians.

A second challenge of the digital health space is that very few are truly accessible, editable, and reusable. The hallmark of science is reproducibility and the simplicity of sharing app code should make this highly feasible. However, the vast majority of apps today are scientific black boxes that are not transparent in how they collect data, analyze results, or share insights. Companies rightfully argue that they cannot be asked to give up their intellectual property when asked for such details. Even apps that are open source with their code freely shared on websites like GitHub are challenging to implement and edit without a strong technical background. Academics note that their funding cycles make it impossible to maintain open platforms, especially when frequent and costly app updates are necessary to keep up with technology changes (De Grood et al. 2016). The resulting situation is likely millions of lines of redundant code, replicating the same sensor functions over and over, and a lack of replicable science. Thus, we aimed to create a platform with complete transparency by using open source code from existing projects where possible and ensuring our focus remained on using the app platform as a mechanism to facilitate innovative research and care. A tangible step in this direction was our reliance on the easily accessible and free resources offered by Open mHealth (“Open mHealth" 2019).

A third challenge lies in using app platforms to explore new data. While many are able to collect active data (surveys) and passive data (sensors), fewer offer metadata. Metadata may be described as data related to how a person completes a survey or cognitive test (e.g., time on each question or target). Sometimes referred to as 'digital exhaust,' this metadata offers advantages of passive data, such as high volume of automatic data collection, but with less ethical challenges (e.g., tap time compared to passive data of GPS coordinates of a person's home) and more clinical relevance and actionability (e.g., knowing someone spent $1 \mathrm{~min}$ to answer a single question about suicide is a clear indication to further explore). For 
illnesses like schizophrenia, where impaired cognition is a primary source of disability (Tandon et al. 2010), and others like depression, where cognitive deficits are often present but overlooked (Gotlib and Joormann 2010), the potential of monitoring cognition via digital device is appealing. Numerous studies in mental health with participants using smartphones have collected active and passive data, but research has paid less attention to metadata and even less on cognition (Moore et al. 2017). Thus, capturing metadata is a focus of our efforts and is therefore reflected in the design of the LAMP platform.

\section{Unmet Needs for Patient Perspectives}

While the needs for app platforms from a clinical and research perspective evolve, patients and users have equally pressing needs for these new digital tools. Using guided survey research (Torous et al. 2018), focus groups, structured interviews, and clinical experience with apps in the mental health care settings, we sought early and continuous input from patients on the platform. It became clear that, just as no one therapy or medication works for all patients, no one app will ever meet all needs. But across diverse patient groups and settings, we noted from three core themes present at all stages of the app development: (1) trust, (2) control, and (3) community.

First, patients explained a need for trust. They wanted to ensure that their data remained under their control and would only be accessed by them, their healthcare team, or the clinical study team, which is consistent with prior research (Di Matteo et al. 2018). Violations of trust, such as the recent experiences from the highly visible Cambridge Analytica to the less visible world of data brokers selling patient data (Glenn and Monteith 2014), are thus important to acknowledge and design against. Desired features to promote trust included both the ability to delete data directly from the app at any time and easily remove the app from the phone if desired. Patients also wanted assurance that their data would be kept secure and confidential.

Second, patients noted that they wanted to be in control of the app, including the ability to customize it to their goals and preferences and not feel surveilled or monitored without consent. This need is consistent with prior qualitative and survey research regarding patient perspectives on mental health apps (de Alva et al. 2015; Ancker et al. 2015). Desired features included an ability to customize questions/surveys, control what sensor data is collected, alter the frequency of all assessments/pop-up reminders, and change the color and language of the app.

Community was the third theme and refers to the patient's concern that the app not isolate them or their care. Rather, patients report their wish for a tool that may be used to strengthen their relationship with their clinical team. They want to connect and share experiences with others while contributing data to science. Prior research findings overwhelmingly assert that apps alone may not provide sufficient emotional support (de Alva et al. 2015). Therefore, future shared decision-making research in digital health may improve patient management of health and personal relationships.

\section{LAMP Overview}

The intersection of these three themes of (1) trust, (2) control, and (3) community with the need for digital health tools to be (1) transparent, (2) data driven, and (3) translational guided the development of the app in our ongoing study, and future clinical plans as outlined in Fig. 1.

The initial design and piloting of the platform occurred over a one-and-a-half-year period, (supported by a grant from the Natalia Mental Health Foundation to MSK and JT and a BIDMC Department of Neurology grant to CL and JT). We utilized multiple iterations of the patient facing app called
Fig. 1 Intersection of patient input and clinical input guiding app design, study, and use

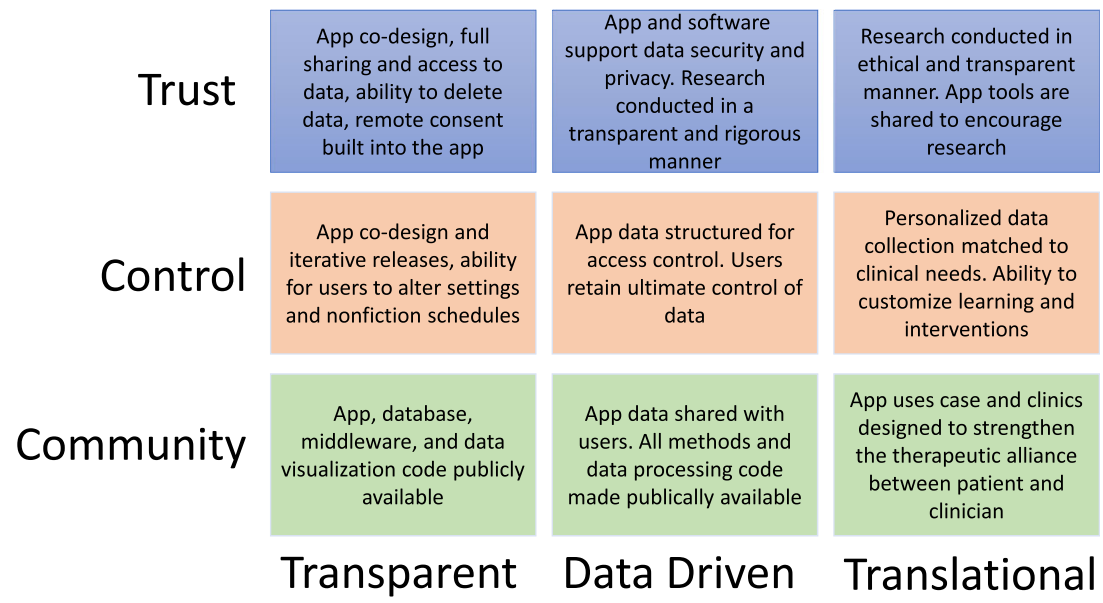


LAMP (Android and Apple) database, portals, and middleware software. During this process, we worked closely with a custom software developer, Zco Corporation, to iterate on the app's concept and build out its various functions. Here, we present the current functional version with the goal of explaining how we balanced clinical, research, and patient needs.

We named the app LAMP, which is an acronym for "Learn," "Assess," "Manage," and "Prevent," to embody the key shared goals of patients and clinicians as well as the translational nature of the platform. The flexibility in use comes from the customizability of each of the four features of the app. The app is customized for each research study via a portal. Data can be accessed from the app itself or the portal as shown in Fig. 2 .

LAMP currently offers a suite of features for both sensor and data collection as well as tools for symptom management and usability. The L-"Learn" tab is a way to provide app users with relevant articles and tips regarding their disorder. These are customizable by every study or clinician and can be uploaded, edited, and updated via a website portal. The $\mathrm{L}$ tab also includes information about the app and what the collected data is used for. The A-" Assess" tab has been a current focus of development for initial studies and thus contains more features. This A tab can be divided into active data (e.g., surveys, cognitive tests, and self-tagging of social/physical space) and passive data (e.g., GPS and fitness data, metadata about screen taps, and the ability to integrate more sensors in the future). Figure 3 offers a schematic of different data streams that can be customized to each study. The goal is to capture and assess symptoms in the context of both environment such as location and physical activity such as steps or sedentary behavior.
The M-"Manage" tab currently includes one management tool to help users focus on core symptom management across many health conditions including sleep, mood, and anxiety as well as specialized ones for schizophrenia including coping with hallucinations. We aim to expand this section by developing more tools with patient input as part of ongoing work. Finally, the P-"Prevent" tab shares data back with users directly on the app and offers gamified tasks to enhance resilience (e.g., mindfulness) and motivate engagement. Selected screenshots featuring the Learn, Assess, Manage, and Prevent tabs of the LAMP app are shown in Fig. 4.

\section{Transparency}

As a research tool, we sought for LAMP to meet the three core principles of the Belmont Report of respect for persons, beneficence, and justice (Belmont Report 1978). But we also sought to ensure the app embodies a transparent approach in use cases outside of research such as clinical care.

Trust is a theme inherently linked to transparency. The iterative design process for creating the app helped build trust as patients witnessed our team's responsiveness to their ideas. Working with our patient advisory panel, focus groups, survey research, and feedback from presentation, we updated the app over ten times in 2018 to ensure that all relevant ideas were implemented. Explaining the mission of LAMP, our commitment to protect their data, and assurance to never sell the app was well received. While all but one current LAMP study involves in-person and face-toface meetings with the research team to help set-up and explain the app, the potential of any app platform also includes remote and scalable use. Thus, at the initial login, we created a quiz feature to help remote users assess their
Fig. 2 The LAMP app can be customized via a portal and resulting data accessed from the app itself or the portal
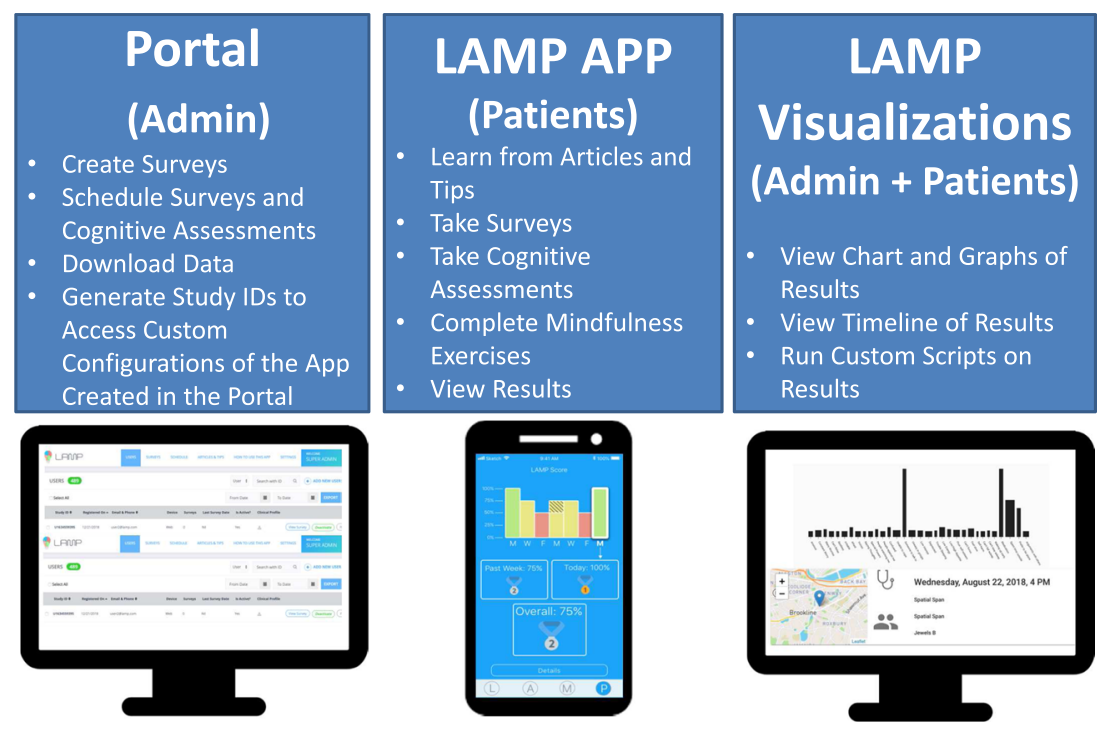
Fig. 3 A schematic of the data capture/assessment features of LAMP

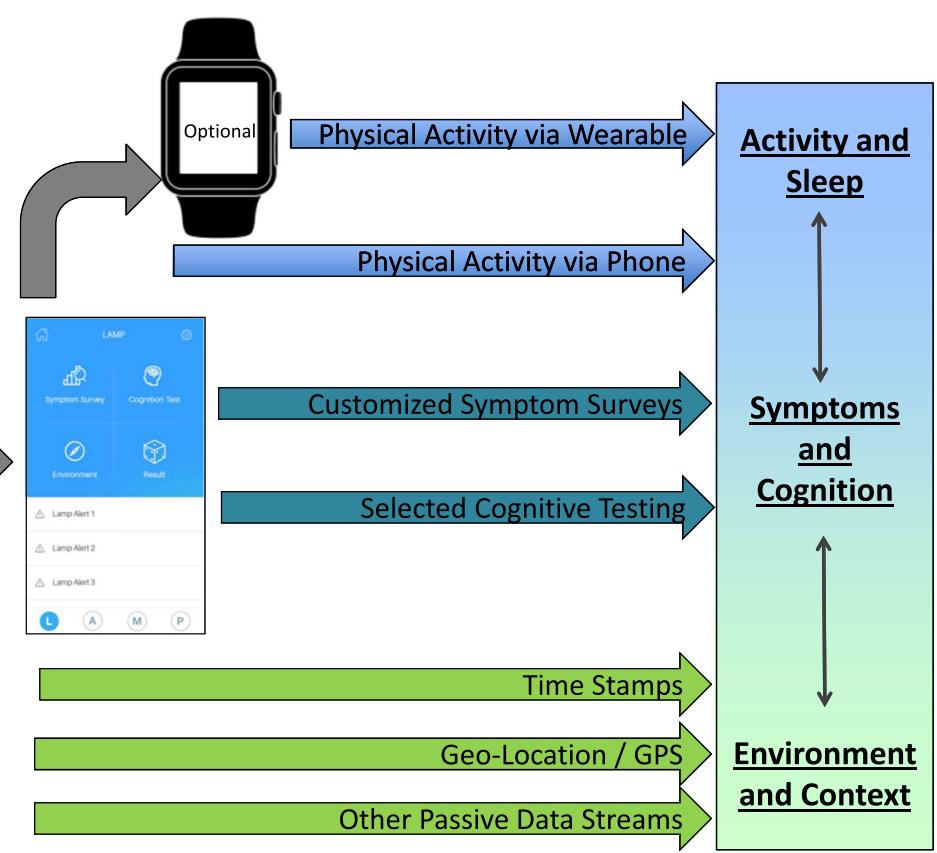

understanding of LAMP and how the app works. Data is owned by the patient or used with consent and permission in approved clinical studies. To further engender trust, we provided the opportunity for patients to review their own
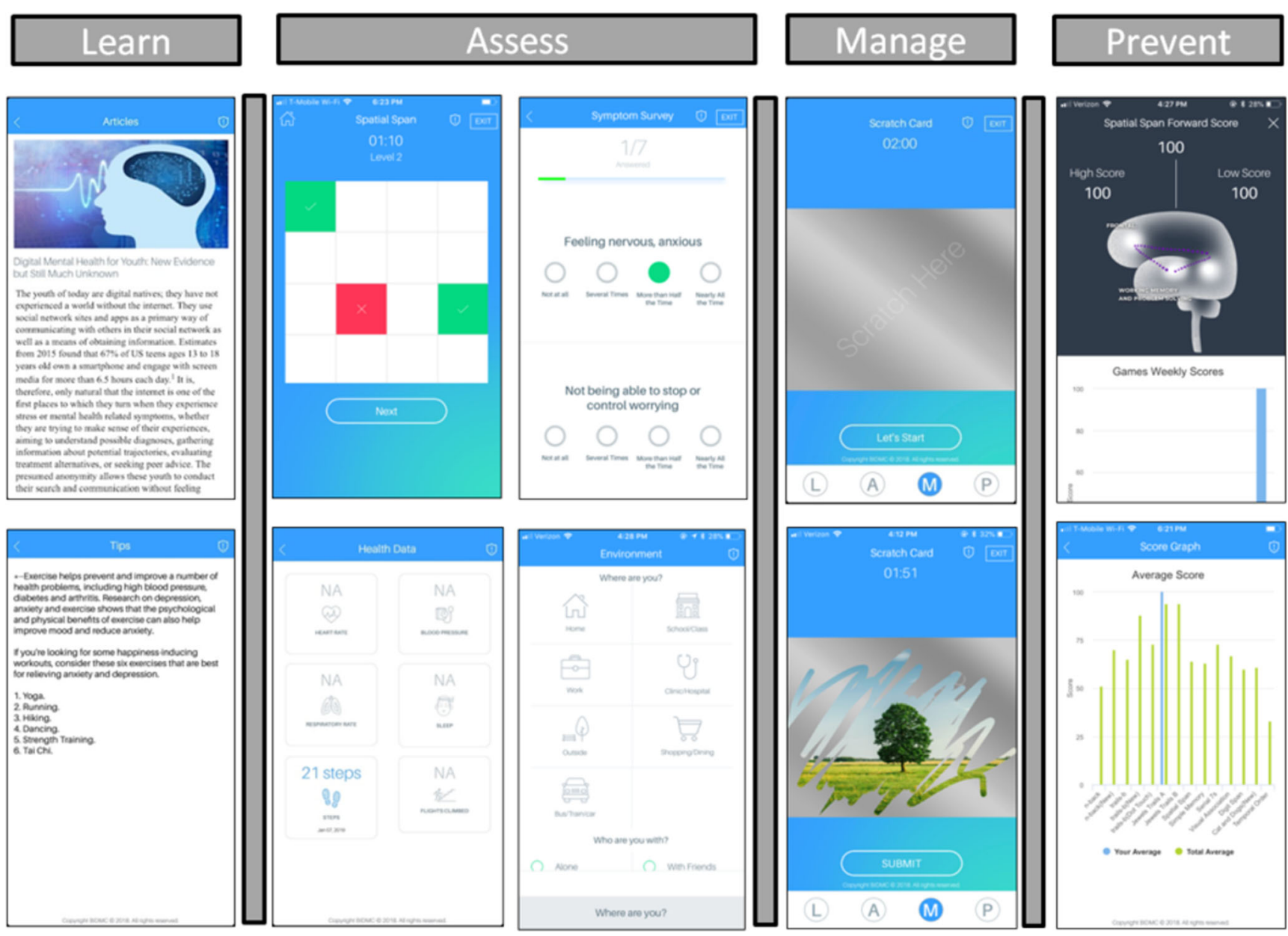

Fig. 4 Screenshots of select features of LAMP across the four domains of Learn, Assess, Manage, and Prevent 
data as well as their ability to signal the research team to delete their data directly from the app. Because it can be challenging to interpret the data collected by the app, we created a visual portal to allow patients to interact with their own data and better understand what is collected.

Control is a theme that we addressed with patients to ensure that LAMP was customizable and adapted to their needs. Many mental health apps, even very popular ones, may not be usable by those with serious mental illnesses like major depression (Sarkar et al. 2016) or schizophrenia spectrum disorders. Working with patients and researchers, we adapted the app to be more engaging and usable. An example of co-design with patients and researchers includes the evolution of the neurocognitive trail-making test from the initial version, where the user is instructed to draw lines on the screen, to a later version without lines, and a current version with jewels replacing circles (see Fig. 5). This not only allows for their control of the app's functionality, but also provides transparency in its development and functions. Other features built into the app allow users to change the background color, set the language from English to either Mandarin, Brazilian Portuguese, or Spanish, change the scheduling of notifications (apart from those scheduled in a study protocol), and send error reports/feedback at any time.

Community was the third guiding theme. Digital health efforts like LAMP involve numerous communities including patients, researchers, software developers, regulators, payers, and other stakeholders. For researchers and software developers, we have posted all of our code for the app, dashboard, and database online for free and publically at https://amp. digital/and shared under an Open Source License. While others can access LAMP, it currently offers most value to researchers given its nascency. Thus, to expand, we have worked to ensure that the software developer community can easily access, edit, and augment LAMP. By creating an application program interface (API), it is easy for others to copy, adapt, connect, and modify our code to augment our version or create their own (Prlić and Procter 2012). Finally, to engage the clinician communities, we have created dashboards that help summarize clinical data in a manner that may offer value in a patient appointment. We are currently working to implement a patient portal where users can access their data in a customized format to suit their needs and interest.

\section{Data Driven}

Another important goal of LAMP is to collect meaningful data. While many apps are able to collect novel types of data including active, passive, and metadata, we sought to futureproof LAMP by also allowing it to easily integrate with other apps and devices like the Apple Watch and Android Google Fit.
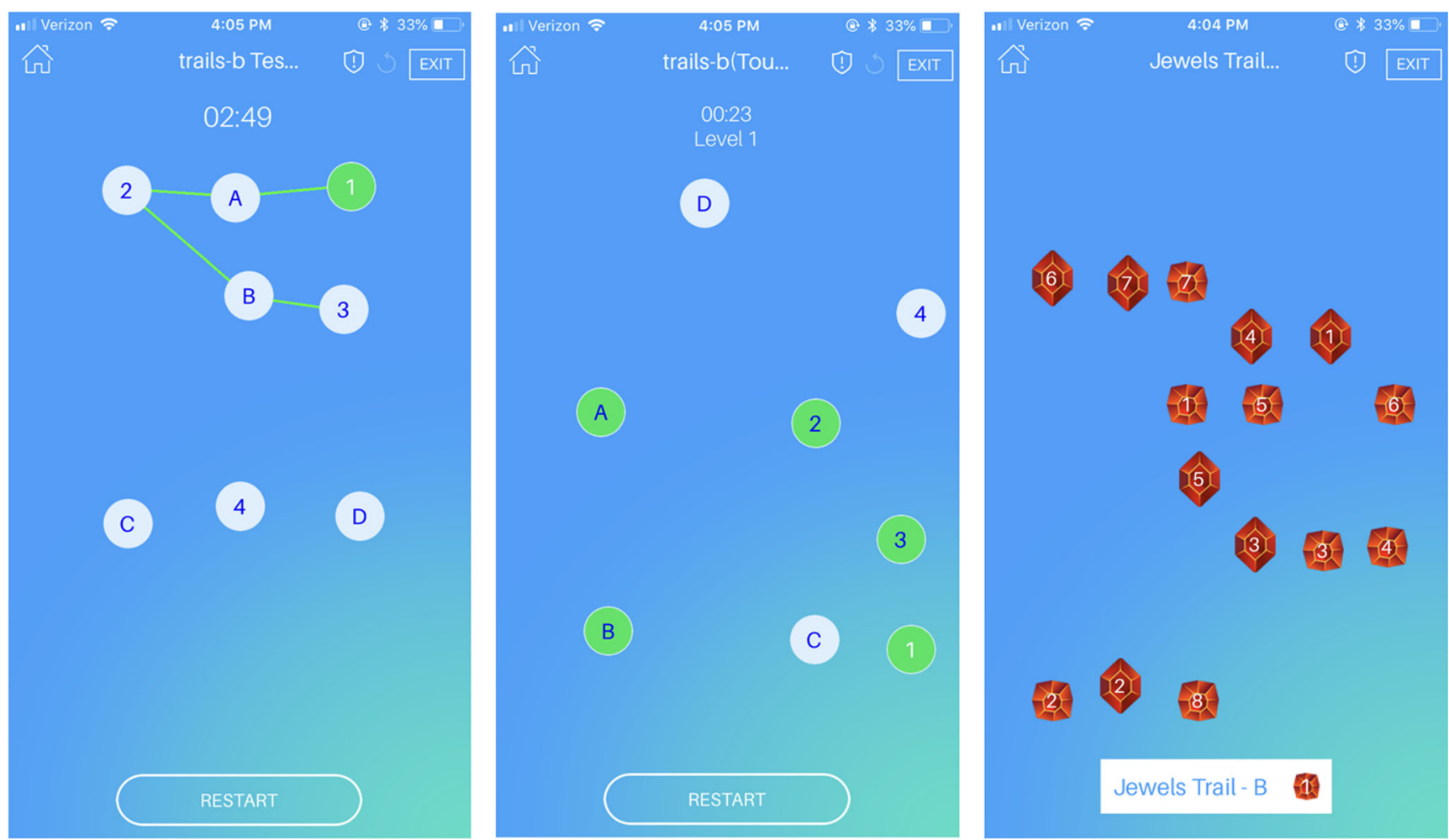

Fig. 5 Adaption of a modified trail test from connecting the dots (left), to tapping the dots (middle) to tapping jewels where the shape now determines order (right) 
Trust is critical for any new data collection tool in healthcare, especially new digital data streams. Given that many mobile app studies correlating sensors to metrics like mood have failed to replicate (Asselbergs et al. 2016; Rohani et al. 2018), we are cautious to make any claims about this data. Instead, our team is committed to building trust through conducting rigorous, fully reproducible research on all LAMP data streams including surveys, sensors, and cognitive tests. Trust in this digital work also involves technical measures to protect data. LAMP uses industry standard encryption protocols that protects data even if the phone is lost. Data is encrypted in transit and on a secure server and we utilize new technologies like docker containers to prevent state leakage as an example of continuous efforts to integrate new data security features.

Control of data is as important as security. By placing the user in control of the data, we ensure that the right control is with the right person. A user can use the app but decide not to grant it access to certain data streams such as GPS or fitness information. As mentioned above, a user can ask that all their data be deleted at any time directly from the app, offering a high level of control over data. We also designed the data structures of LAMP to reflect proper control of the data as shown in Fig. 6. In this research configuration, a researcher has access to one or more studies, and each of those studies has access to one or more participants, who have access to one or more types of data the app has collected. By building this hierarchy into the app data, the organization of the data supports control by its very structure.

Community regarding LAMP data means using the right data to support the right relationships. Focusing first on the data, we share Jupyter Notebooks that contain our code for processing and visualizing the data (https://lamp.digital/). For researchers, we created a dashboard that offers the ability to analyze data in $\mathrm{R}$ or Python by running scripts either directly on study data in the cloud or on downloadable datasets. By making these resources available to the community, we hope advances in methods will develop for translating raw data into clinically meaningful insights. While sharing patient-specific data is often not permissible or ethical, we designed the data structure in LAMP for scalability and interoperability. The system can be used in multiple research studies with the same expected output, regardless of input data.

Figure 7 demonstrates how different activities in a cognition and symptom monitoring study can be uploaded and compiled together in a clear, concise format. Thus, as others add custom surveys to capture data that matters to them and add novel cognitive tests, the same underlying data structure can be utilized and ensures data compatibility even with older versions and assessments. With these community features in place, we hope LAMP data will offer a vibrant community for researchers and developers. We acknowledge that we have not yet focused on using LAMP data to form a patient-focused community that could, for example, share such data towards fostering peer support or matching patients with those who have similar experiences as their own. As the initial pilot studies of the app conclude, we plan to reach out to local patient organizations to explore new use cases and new features. Given that social support is one of the key drivers of app engagement (Torous et al. 2018b), patient communities will be necessary to lead that effort.

We have also created and posted an application program interface (API) (https://lamp.digital/) which makes it easy for others to work with LAMP data in a standardized way and integrate it into their own app. While we are still developing and adding to LAMP, we believe making it available in its current state allows others to replicate our work, advance upon it, develop new use cases, and innovate to their

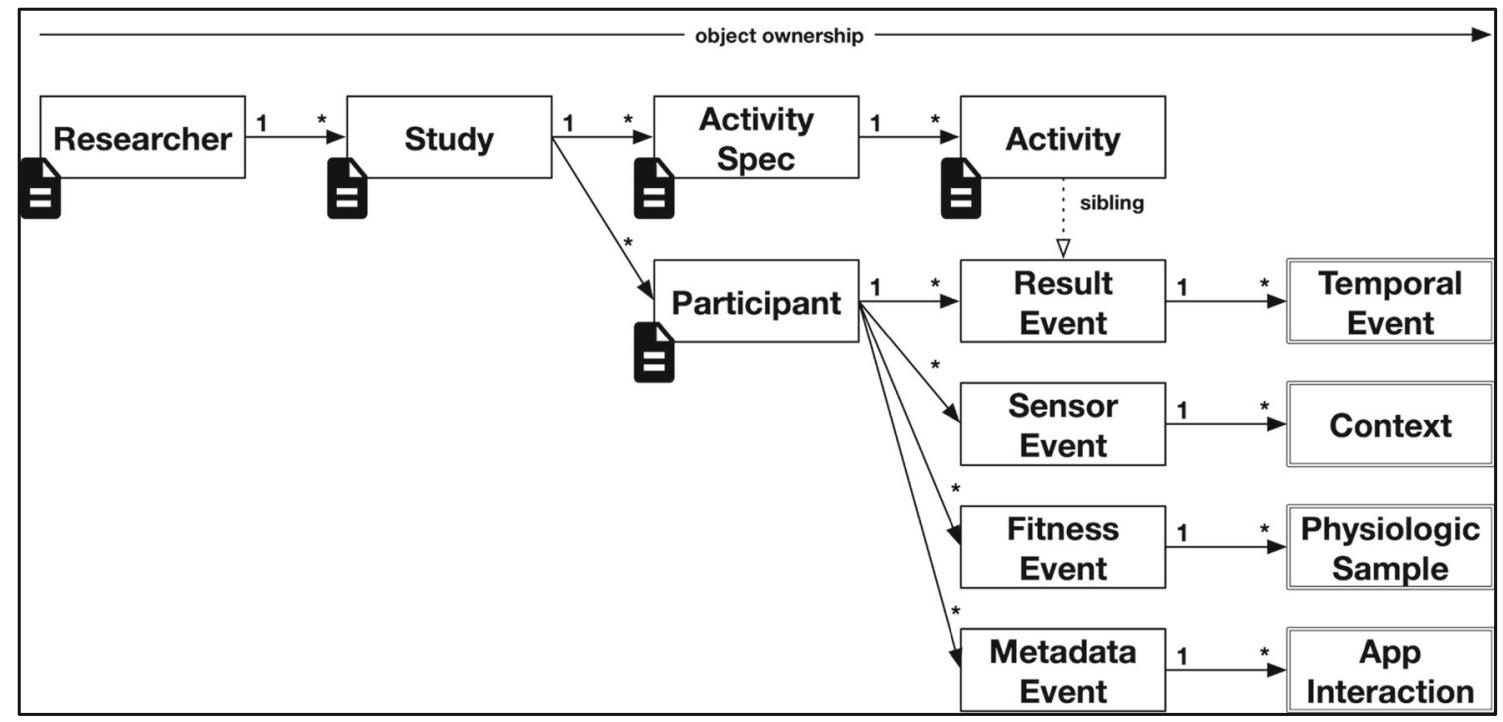

Fig. 6 Data types and hierarchy for LAMP data 


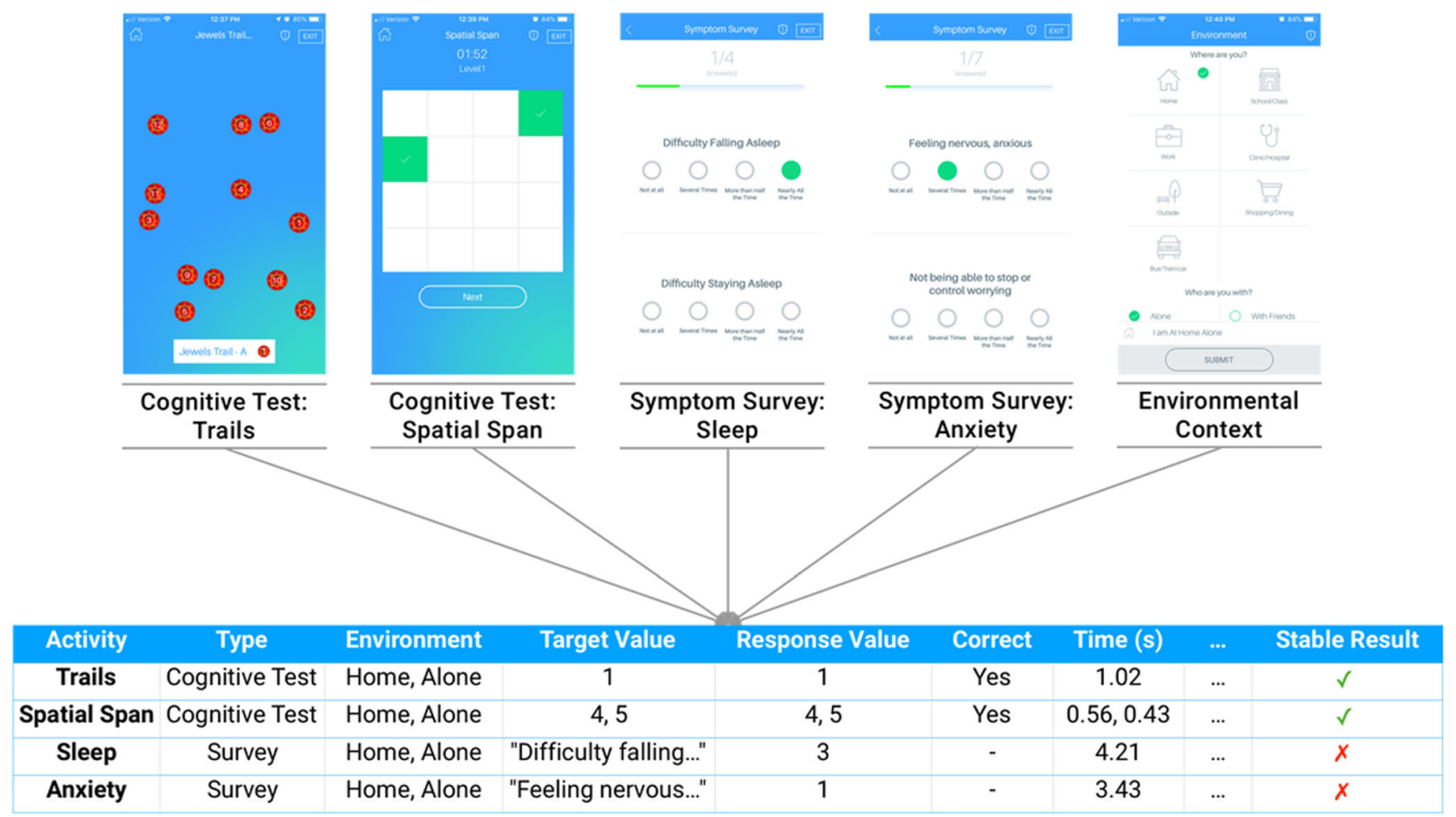

Fig. 7 The unified data structure of LAMP is designed to facilitate analysis of assessment data

customized needs. Thus, we believe the project can keep pace with the rapid advances and innovations in digital health.

\section{Translational}

While the data generated by LAMP, and other health apps, is useful, we believe the true potential of smartphone apps is their ability to serve as a translational device to improve clinical outcomes. We designed LAMP as a tool that both patients and clinicians will use together in diagnostic assessments, monitoring, and interventions. We explicitly refer to LAMP as a tool since the potential of the app comes not from the underlying software, but rather the people using it.

Establishing trust for any new clinical tool is a process that relies on reproducible research findings and clinical utility. Thus, our team is embarking on several LAMP studies at this time as well as sharing the app with diverse teams. For example, researchers in an anesthesiology department (JP and AT) are investigating the L-"Learn" features of LAMP in a study to educate patients about perioperative surgery recovery and anesthesia. A research team in China is focusing on A-"Assess" with ongoing research to analyze the cognitive data assessed by the app and if it may help predict conversion to schizophrenia from the prodrome. Researchers at Boston University (DF and EW) are assessing the relationship between fluctuations in cognition and factors such as mood, motivation, and social isolation. In collaboration with a research team in Canada (led by SG), the Beth Israel Deaconess Medical Center psychiatry team (JT, HW, PH, AV, MK) is conducting an ongoing study that aims to assess cognitive data gathered from LAMP and compare them to gold standard measures in depression or schizophrenia. The Beth Israel Deaconess Medical Center neurology team (CL, HT) is studying whether cognitive symptoms and metadata from LAMP may correlate with clinical decline in patients with early symptoms of Alzheimer's disease.

As a tool, LAMP was designed to offer control to patients and clinicians. We envision use cases where the clinician and patient can together determine what they may wish to track - be it physical activity, cognition, custom survey questions, or social engagement - and quickly customize LAMP to collect only the data necessary and clinically relevant. Likewise, as more interventions are built out by various teams, a range of digital interventions will be available for selection as needed. The initial intervention in LAMP was informed by patient experience at Cambridge Health Alliance, a large primary care/hospital system where mental health clinicians share mobile app resources with patients to augment and extend in-person clinical services (see 'Assess' in Fig. 4).

The goal is not to use all the features of LAMP but instead use the right ones for the right patient. Towards this goal, we are piloting a digital mental health clinic to 
better understand what is the right way to help patients and clinicians customize the app in the most clinically effective manner. We have already created a suite of data visualization software to help patients and clinicians understand their data and assess the value of information collection (see Fig. 8). While data visualization of digital health data remains a nascent area, good visual representations can spark a clinically relevant conversation and allow new insights (Torous and Roux 2017).

Community is central to LAMP and reflects using the app to strengthen the therapeutic alliance between patients and clinicians. While technology may help patients with self-management, the low engagement rates with most self-help apps speak to the challenges for stand-alone apps (Mueller et al. 2018). LAMP includes traditional elements to augment engagement such as awarding points for completion of app activities and spinning a wheel to win prizes (see Fig. 9 below). Clinician engagement is also a challenge, especially at a time where burnout is increasingly associated with excessive technology use related to electronic medical records (Domaney et al. 2018). One of the chief reasons for failure of digital health tools remains low uptake on the clinical side (Gagnon et al. 2015), but by using LAMP as a tool not to isolate patients or clinicians, but rather to strengthen their alliance, we see a brighter potential for LAMP.
Fig. 8 A screenshot of sample LAMP data, created by for sharing using no clinical data, visualized on the web portal

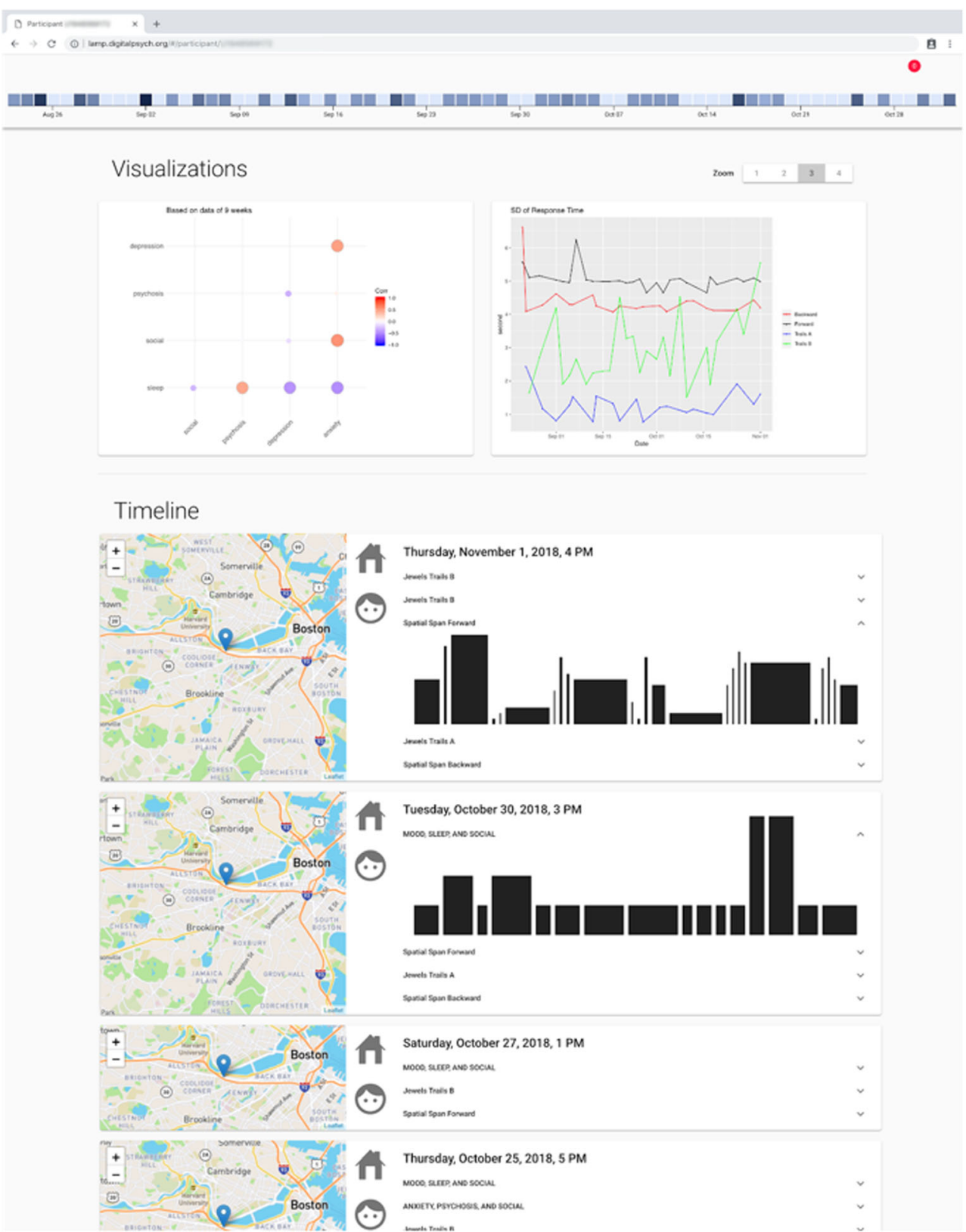


Fig. 9 Gamified elements of LAMP
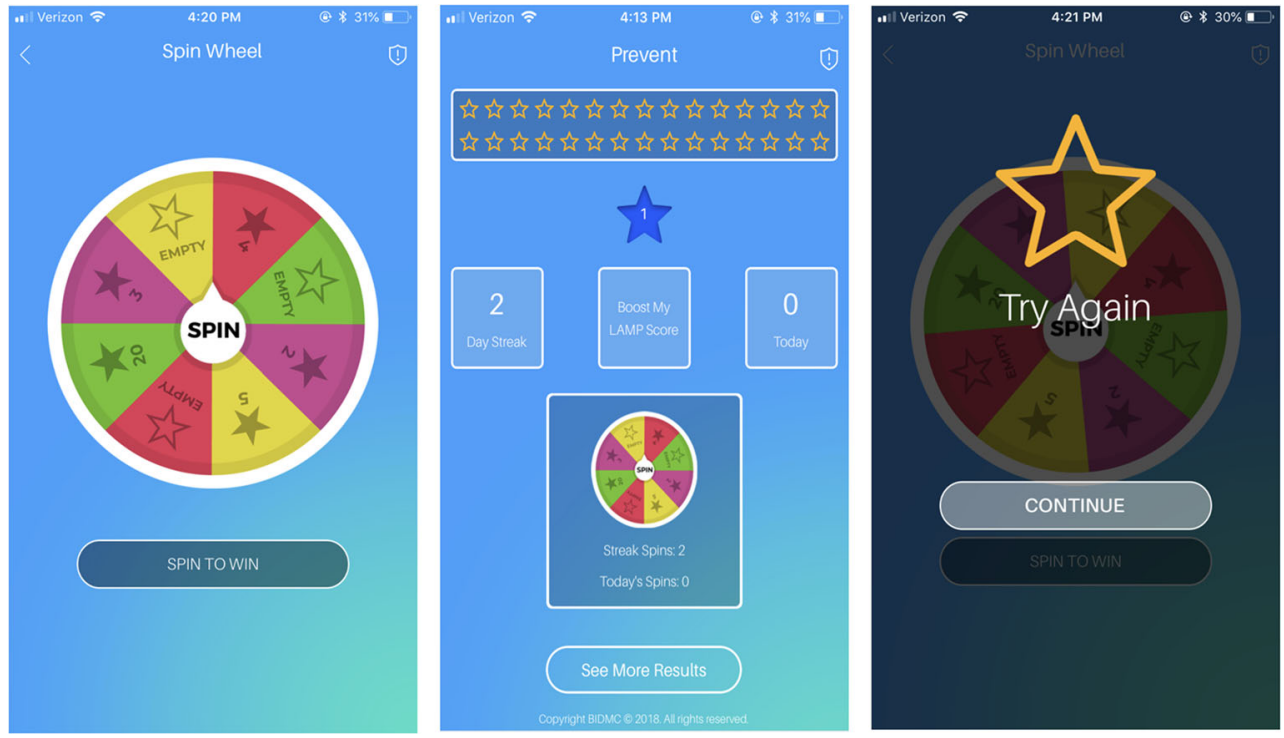

\section{Future Directions for LAMP}

\section{Digital Psychiatry Clinic}

At the digital psychiatry clinic starting in March 2019, we will use LAMP to augmented care as shown below in Fig. 10. To not overburden either the patient or clinician with issues around technology in the appointment (Hilty et al. 2018b), the digital clinic will employ a new staff member: a digital navigator. While the concept of a digital navigator is relatively new (Ben-Zeev et al. 2015), the digital clinic for mental health is even more nascent. The digital navigator will serve as a liaison between the digital data and information generated by the app, on demand support for any technology set up or issues, and the team member responsible for remote monitoring of the data. With further experience, the role may be modified but the initial iteration of the digital clinic will feature LAMP and the digital navigator in these roles that are intended to support a strong therapeutic alliance where it matters the most. As digital clinics evolve and build evidence, practice models and competencies will be a next step in dissemination (Hilty et al. 2019).
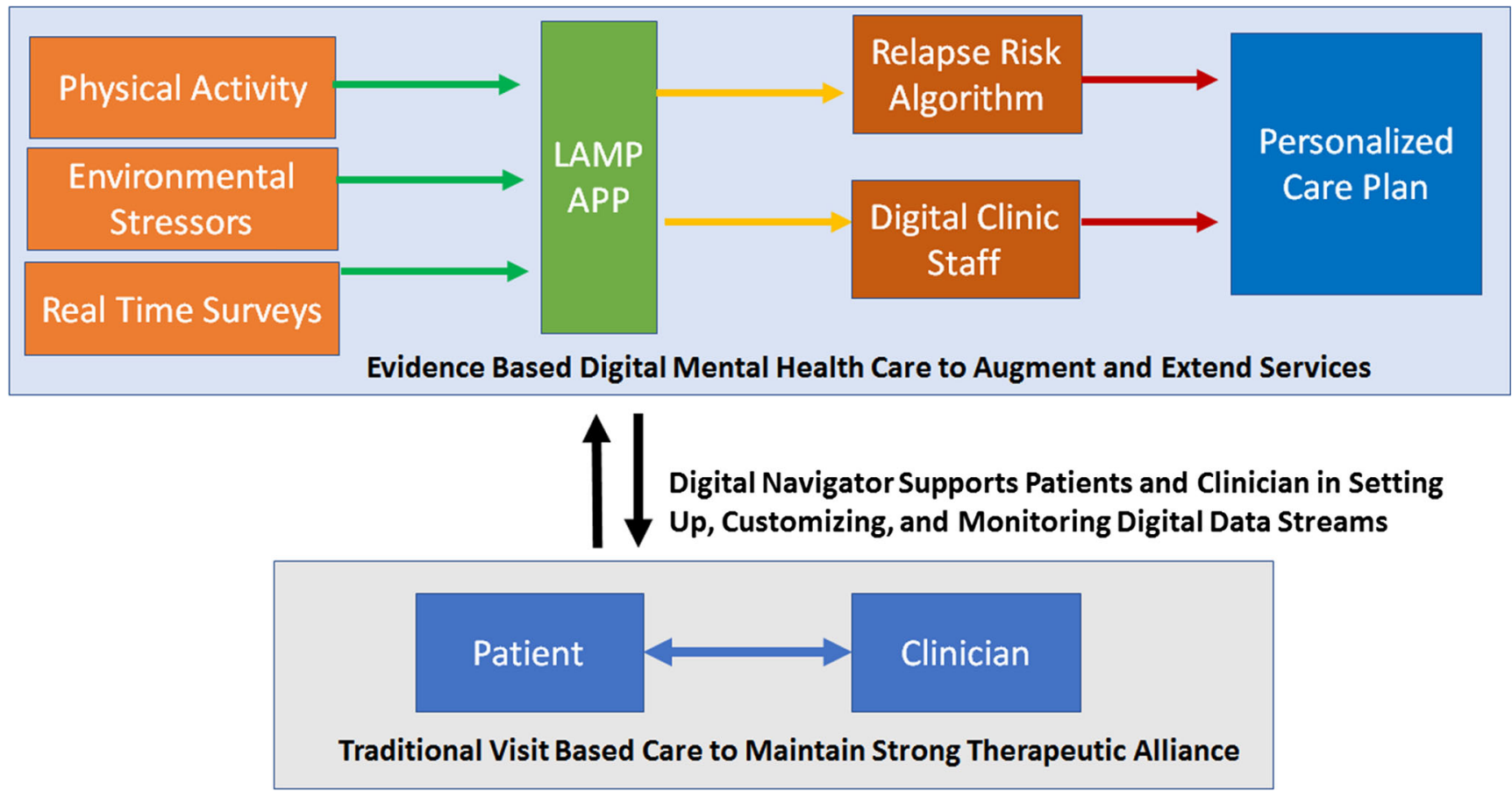

Fig. 10 Schematic of the Digital Clinic 


\section{Youth Mental Health}

Use of LAMP via a digital clinic has high potential for care in both youth mental health and underserved patient groups. Fewer than half of young people have access to evidence-based mental health care, including high-income countries such as the USA (Patel et al. 2018). With the onset of nearly three quarters of mental disorders occurring before age 24 (Kessler et al. 2005), there may be unique opportunities to leverage this increasing use of digital technology for detecting risk factors for mental illness, facilitating referral, and delivering targeted digital interventions for these young individuals. The application of digital phenotyping early in life could yield necessary insights to understand risk factors for mental illness, as well as the precise timing of the onset of problematic symptoms earlier than existing clinical diagnostic approaches.

\section{Underserved Patient Groups}

There is considerable evidence supporting the feasibility, acceptability, and initial clinical effectiveness of digital mental health interventions across diverse low- and middle-income countries (Naslund et al. 2017). Use of LAMP to capture real-time assessments and signals to create a user's digital phenotype could trigger the delivery of other digital resources directly through the smartphone platform to support an at-risk individual. This could include various forms of immediate support tailored to an individual's needs at that moment, such as preventing relapse, offering self-help activities, or motivation to overcome problem behaviors or low mood. Furthermore, the digital platform could send real-time alerts to nonspecialist health providers, such as community health workers or other lay providers, who make up the predominant health care workforce in many lower resource settings. This direct connection to a non-specialist health provider can support the integration of the digital platform into the existing health system. The true potential for open source and shareable digital platforms such as LAMP may be realized in low-resource settings, where health systems are heavily under-resourced and fragmented, and where the demand for evidence-based mental health services is greatest.

\section{Conclusion}

The Institute of Medicine defines a learning healthcare system as one in which "science, informatics, incentives, and culture are aligned for continuous improvement and innovation, with best practices seamlessly embedded in the delivery process and new knowledge captured as an integral by-product of the delivery experience (McGinnis et al. 2007). Just like a learning healthcare system is itself a sociotechnical system that relies on new technologies to help gather and analyze data and multidisciplinary teams to implement change, we view LAMP as a sociotechnical system that relies on both technology and people. The healthcare system will continue to adapt, as will the technology and capabilities of apps, but the intersection between patient needs for digital health (i.e., trust, control, community) and clinical research needs for digital health (i.e., transparent, data driven, translational) will likely remain constant. The intersection of diverse individuals and teams who helped create LAMP embodies the synergies and collaborative advancements we hope LAMP may now share with others. Realizing there is always room for improvement and more perspectives, we hope others will adapt, expand, and augment LAMP to meet the needs of their own healthcare systems, clinicians, and patients.

Funding Information This project was supported by a generous donation from the Natalia Mental Health Foundation, a departmental award from the BIDMC Department of Neurology, and support from the BIDMC Department of Psychiatry.

Compliance with Ethical Standards This study was reviewed and approved by the Institutional Review Board and all participants gave their informed consent prior to the start of the study. All data on phones, on the server where the data is stored, and in-transit use industry-standard encryption techniques.

Conflict of Interest The authors declare that they have no conflict of interest.

Open Access This article is distributed under the terms of the Creative Commons Attribution 4.0 International License (http:// creativecommons.org/licenses/by/4.0/), which permits unrestricted use, distribution, and reproduction in any medium, provided you give appropriate credit to the original author(s) and the source, provide a link to the Creative Commons license, and indicate if changes were made.

\section{References}

Ancker, J. S., Witteman, H. O., Hafeez, B., Provencher, T., Van de Graaf, M., \& Wei, E. (2015). "You get reminded you're a sick person": Personal data tracking and patients with multiple chronic conditions. Journal of Medical Internet Research, 17(8).

Anthes, E. (2016). Mental health: there's an app for that. Nature News, 532(7597), 20.

Asselbergs, J., Ruwaard, J., Ejdys, M., Schrader, N., Sijbrandij, M., \& Riper, H. (2016). mMobile phone-based unobtrusive ecological momentary assessment of day-to-day mood: an explorative study. Journal of Medical Internet Research., 18(3).

Ben-Zeev, D., Drake, R., \& Marsch, L. (2015). Clinical technology specialists. BMJ: British Medical Journal (Online), 350.

Bhugra, D., Tasman, A., Pathare, S., Priebe, S., Smith, S., Torous, J., et al. (2017). The WPA-lancet psychiatry commission on the future of psychiatry. The Lancet Psychiatry, 4(10), 775-818. 
de Alva, F. E. M., Wadley, G., \& Lederman, R. (2015). It feels different from real life: Users' Opinions of Mobile Applications for Mental Health. In Proceedings of the Annual Meeting of the Australian Special Interest Group for Computer Human Interaction (pp. 598602). ACM

De Grood, C., Raissi, A., Kwon, Y., \& Santana, M. J. (2016). Adoption of e-health technology by physicians: a scoping review. Journal of Multidisciplinary Healthcare, 9, 335.

Di Matteo, D., Fine, A., Fotinos, K., Rose, J., \& Katzman, M. (2018). Patient willingness to consent to mobile phone data collection for mental health apps: structured questionnaire. JMIR Mental Health, $5(3)$.

Domaney, N. M., Torous, J., \& Greenberg, W. E. (2018). Exploring the association between electronic health record use and burnout among psychiatry residents and faculty: a pilot survey study. Academic Psychiatry, 1-5.

Firth, J., Torous, J., \& Yung, A. R. (2016). Ecological momentary assessment and beyond: the rising interest in e-mental health research. Journal of Psychiatric Research, 80, 3-4.

Firth, J., Torous, J., Nicholas, J., Carney, R., Pratap, A., Rosenbaum, S., \& Sarris, J. (2017a). The efficacy of smartphone-based mental health interventions for depressive symptoms: a meta-analysis of randomized controlled trials. World Psychiatry, 16(3), 287-298.

Firth, J., Torous, J., Nicholas, J., Carney, R., Rosenbaum, S., \& Sarris, J. (2017b). Can smartphone mental health interventions reduce symptoms of anxiety? A meta-analysis of randomized controlled trials. Journal of affective disorders, 218, 15-22.

Fleming, T., Bavin, L., Lucassen, M., Stasiak, K., Hopkins, S., \& Merry, S. (2018). Beyond the trial: systematic review of real-world uptake and engagement with digital self-help interventions for depression, low mood, or anxiety. Journal of Medical Internet Research, 20(6), e199.

Gagnon, M. P., Ngangue, P., \& Payne-Gagnon, J. (2015). Desmartis M. $\mathrm{m}$-Health adoption by healthcare professionals: a systematic review. Journal of the American Medical Informatics Association., 23(1), 212-220.

Glenn, T., \& Monteith, S. (2014). Privacy in the digital world: medical and health data outside of HIPAA protections. Current Psychiatry Reports, 16(11), 494.

Gotlib, I. H., \& Joormann, J. (2010). Cognition and depression: current status and future directions. Annual Review of Clinical Psychology., 6, 285-312.

Hilty, D. M., Chan, S., Hwang, T., Wong, A., \& Bauer, A. M. (2017). Advances in mobile mental health: opportunities and implications for the spectrum of e-mental health services. Mhealth, 3 .

Hilty, D. M., Maheu, M. M., Drude, K. P., \& Hertlein, K. M. (2018a). The need to implement and evaluate telehealth competency frameworks to ensure quality care across behavioral health professions. Academic Psychiatry, 42(6), 818-824.

Hilty, D. M., Turvey, C., \& Hwang, T. (2018b). Lifelong learning for clinical practice: how to leverage technology for telebehavioral health care and digital continuing medical education. Current Psychiatry Reports, 20(3), 15.

Hilty, D. M., Sunderji, N., Suo, S., Chan, S., \& McCarron, R. M. (2019). Telepsychiatry/telebehavioral health and integrated care: evidencebase, best practice models and competencies. International Review of Psychiatry, In Press. https://doi.org/10.1080/09540261.2019. 1571483.

Hsin, H., \& Torous, J. (2018). Creating boundaries to empower digital health technology. BJPsych Open, 4(4), 235-237.

Kessler, R. C., Berglund, P., Demler, O., Jin, R., Merikangas, K. R., \& Walters, E. E. (2005). Lifetime prevalence and age-of-onset distributions of DSM-IV disorders in the National Comorbidity Survey Replication. Archives of General Psychiatry., 62(6), 593-602.

McGinnis, J. M., Aisner, D., \& Olsen, L. (Eds.). (2007). The learning healthcare system: workshop summary. National Academies Press.
McNiel, D. E., \& Binder, R. (2018). Current regulation of mobile mental health applications. The Journal of the American Academy of Psychiatry and the Law, 46, 204-211.

Mobile phone based sensing software. (2019, January 22). Retrieved from https://en.wikipedia.org/wiki/Mobile_phone_based_sensing software

Mohr, D. C., Zhang, M., \& Schueller, S. M. (2017). Personal sensing: understanding mental health using ubiquitous sensors and machine learning. Annual Review of Clinical Psychology, 13, 23-47.

Moore, R. C., Swendsen, J., \& Depp, C. A. (2017). Applications for selfadministered mobile cognitive assessments in clinical research: a systematic review. International Journal of Methods in Psychiatric Research, 26(4), e1562.

Mueller, N. E., Panch, T., Macias, C., Cohen, B. M., Ongur, D., \& Baker, J. T. (2018). Using smartphone apps to promote psychiatric rehabilitation in a peer-led community support program: pilot study. JMIR Mental Health, 5(3).

Naslund, J. A., Aschbrenner, K. A., Araya, R., et al. (2017). Digital technology for treating and preventing mental disorders in lowincome and middle-income countries: a narrative review of the literature. The Lancet Psychiatry., 4(6), 486-500.

National Commission for the Protection of Human Subjects of Biomedical and Behavioral Research (1978). The Belmont report: Ethical principles and guidelines for the protection of human subjects of research. [Bethesda, Md.]: The Commission.

Nicholas, J., Larsen, M. E., Proudfoot, J., \& Christensen, H. (2015). Mobile apps for bipolar disorder: a systematic review of features and content quality. Journal of Medical Internet Research, 17(8).

Noel, V. A., Acquilano, S. C., Carpenter-Song, E., \& Drake, R. E. (2018). Assessing people with serious mental illness' use of mobile and computer devices to support recovery. JMIR Ment Health. https:// doi.org/10.2196/12255.

"Open mHealth". (2019, January 22). Retrieved from http://www. openmhealth.org/

Patel, et al. (2018). The Lancet Commission on global mental health and sustainable development. The Lancet.

Prlić, A., \& Procter, J. B. (2012). Ten simple rules for the open development of scientific software. PLoS Computational Biology, 8(12), e1002802.

Rohani, D. A., Faurholt-Jepsen, M., Kessing, L. V., \& Bardram, J. E. (2018). Correlations between objective behavioral features collected from mobile and wearable devices and depressive mood symptoms in patients with affective disorders: systematic review. JMIR mHealth and uHealth, 6(8).

Safavi, K., Mathews, S. C., Bates, D. W., Dorsey, E. R., \& Cohen, A. B. (2019). Top-funded digital health companies and their impact on high-burden, high-cost conditions. Health Affairs, 38(1), 115-123.

Sarkar, U., Gourley, G. I., Lyles, C. R., Tieu, L., Clarity, C., Newmark, L., et al. (2016). Usability of commercially available mobile applications for diverse patients. Journal of General Internal Medicine, 31(12), 1417-1426.

Schueller, S. M., Washburn, J. J., \& Price, M. (2016). Exploring mental health providers' interest in using web and mobile-based tools in their practices. Internet Interventions, 4, 145-151.

Tandon, R., Nasrallah, H. A., \& Keshavan, M. S. (2010). Schizophrenia, "Just the Facts" 5. Treatment and prevention Past, present, and future. Schizophrenia Research, 122(1-3), 1-23.

Torous, J., \& Haim, A. (2018). Dichotomies in the Development and Implementation of Digital Mental Health Tools. Psychiatric Services, 69(12), 1204-1206.

Torous, J., \& Roberts, L. W. (2017). Needed innovation in digital health and smartphone applications for mental health: transparency and trust. JAMA Psychiatry, 74(5), 437-438.

Torous, J., \& Roux, S. (2017). Patient-driven innovation for mobile mental health technology: case report of symptom tracking in schizophrenia. JMIR Mental Health, 4(3). 
Torous, J., Kiang, M. V., Lorme, J., \& Onnela, J. P. (2016). New tools for new research in psychiatry: a scalable and customizable platform to empower data driven smartphone research. JMIR Mental Health, $3(2)$.

Torous, J., Wisniewski, H., Liu, G., \& Keshavan, M. (2018). Mental health mobile phone app usage, concerns, and benefits among psychiatric outpatients: comparative survey study. JMIR Mental Health., 5(4).

Torous, J., Nicholas, J., Larsen, M. E., Firth, J., \& Christensen, H. (2018b). Clinical review of user engagement with mental health smartphone apps: evidence, theory and improvements. EvidenceBased Mental Health, 21(3), 116-119.

United Nations Population Fund (UNFPA). (2014). The power of 1.8 billion: adolescents, youth and the transformation of the future. New York: UNFPA State of World Population 2014.

Publisher's Note Springer Nature remains neutral with regard to jurisdictional claims in published maps and institutional affiliations. 